Revista Med 26(2) 2018

\title{
SÍNDROME DE EAGLE: REPORTE DE CASO
}

\author{
LAURA ESTEFANÍA MONTENEGRO SANTOFIMIO', DANIELA MARULANDA \\ GRAJALES ${ }^{1}$, SERGIO MAURICIO CASTELLANOS GARCÍA ${ }^{2}$ \\ ${ }^{1}$ Residente de Cirugía Oral y Maxilofacial, Servicio de Cirugía Oral y \\ Maxilofacial, Hospital Militar Central, Bogotá, Colombia. \\ ${ }^{2}$ Cirujano Oral y Maxilofacial, Servicio de Cirugía Oral y Maxilofacial, \\ Hospital Militar Central, Bogotá, Colombia. \\ Correspondencia: Daniela Marulanda Grajales \\ danimaru93@gmail.com
}

Recibido: 4 de octubre de 2017 Aceptado: 31 de agosto de 2018

\section{Resumen}

El síndrome de Eagle se considera como la elongación del proceso estiloides con osificación del ligamento estilohioideo o sin él, que se extiende entre el hueso temporal y el hioides. El diagnóstico puede ser realizado principalmente con tomografía computarizada (TC) o radiografía panorámica, por su puesto sin dejar de lado la variedad de signos y síntomas, como dolor cervical, sensación de cuerpo extraño en la faringe, limitaciones en la apertura bucal, dificultad para la fonación, disfagia, otalgia, tinnitus, trismo y movilidad reducida del cuello. Ya identificada la elongación, el paciente podrá recibir un tratamiento conservador o quirúrgico dependiendo de la severidad de los síntomas. El manejo conservador incluye fisioterapia, infiltración con anestésicos locales o corticosteroides y el tratamiento con fármacos antiinflamatorios, anticonvulsivos o antidepresivos. El manejo quirúrgico consiste en fracturar el proceso estiloides bajo anestesia general, a través de abordajes transorales o transcervicales. A continuación, expondremos información pertinente sobre el síndrome de Eagle y posteriormente presentaremos un caso clínico de una paciente con dicho síndrome del servicio de Cirugía Oral y Maxilofacial del Hospital Militar Central.

Palabras clave: síndrome de Eagle; elongación estiloides; calcificación ligamento estilohioideo; dolor facial; neuralgia

\section{EAGLE SYNDROME: CASE REPORT}

\begin{abstract}
Eagle syndrome is considered as the elongation of the styloid process with or without ossification of the stylohyoid ligament, which extends between the temporal bone and the hyoid. The diagnosis can be made mainly with computed tomography (CT) or panoramic radiography, of course without neglecting the variety of signs and symptoms, such as cervical pain, foreign body sensation in the pharynx, limitations in oral opening, difficulty in phonation, dysphagia, otalgia, tinnitus, trismus and reduced neck mobility. Once the elongation has been identified, the patient may receive conservative or surgical treatment depending on the severity of the symptoms. Conservative management includes physiotherapy, infiltration with local anesthetics or corticosteroids and treatment with anti-inflammatory, anticonvul-
\end{abstract}


sant or antidepressant drugs. Surgical management consists in fracturing the styloid process under general anesthesia, through transoral or transcervical approaches. Next, we will present pertinent information about the Eagle syndrome and then we will present a clinical case of a patient with said syndrome of the Oral and Maxillofacial Surgery service of the Hospital Militar Central.

Keywords: Eagle syndrome; styloid elongation; stylohyoid ligament calcification.

\section{SÍNDROME DE EAGLE: RELATO DE CASO}

\section{Resumo}

A sindrome de Eagle considera-se como o alongamento do processo estiloide com ossificação do ligamento estilohioideo ou sem ele, que se estende entre o osso temporal e os hioides. O diagnóstico pode ser realizado principalmente com tomografia computadorizada (TC) ou radiografia panorâmica, obviamente sem deixar de lado a variedade de sinais e sintomas, como dor cervical, sensação de corpo estranho na faringe, limitações na abertura bucal, dificuldade para a fonação, disfagia, otalgia, zumbido, trismo e mobilidade reduzida do pescoço. Já identificado o alongamento, o paciente poderá receber um tratamento conservador ou cirúrgico dependendo da gravidade dos sintomas. O tratamento conservador inclui fisioterapia, infiltração com anestésicos locais ou corticosteroides e o tratamento com fármacos anti-inflamatórios, anticonvulsivos ou antidepressivos. O tratamento cirúrgico consiste em fraturar o processo estiloide sob anestesia geral, por meio de abordagens intraorais ou transcervicais. A seguir, exporemos informação pertinente sobre a síndrome de Eagle e, posteriormente, apresentaremos um caso clínico de uma paciente com essa sindrome do serviço de Cirurgia Oral e Maxilofacial do Hospital Militar Central.

Palavras-chave: síndrome de Eagle; alongamento estiloides; calcificação ligamento estilo-hioideo.

\section{Introducción}

El sindrome de Eagle es una secuela poco frecuente de la elongación del proceso estiloides, es posible que sea responsable de síntomas que pueden imitar otras patologías y manifestarse como dolor en el cuello o dolor referido en la oreja (1).

En 1937, Eagle describió un síndrome asociado con dolor facial que surge del proceso estiloideo y lo clasificó en dos subtipos: síndrome clásico estiloideo y síndrome de la arteria estilocarotidea (2).

El proceso estiloideo es una protuberancia ósea delgada que emerge de la base del cráneo, cuenta con varios anexos que incluyen el foramen estilomastoideo, el foramen yugular, el proceso mastoideo y, lo más importante, el canal carotídeo. Las longitudes del proceso estiloideo típico oscilan entre 1 y $3 \mathrm{~cm}$ y los procesos estiloides de más de $3 \mathrm{~cm}$ se definen como largos (3).

\section{Incidencia}

Eagle en 1937 informó un 4\% de estiramiento del estiloides de la población general, de los cuales solo el $4 \%$ reportan sintomas. Sin embargo, en 1970, Kaufman, en un estudio de 484 pacientes, encontró que la incidencia de la elongación de la estiloides fue mucho mayor, cerca del $7 \%$ (4). En 1924. Guthrie examinó 2000 cráneos humanos $(0,55 \%)$ que presentaron procesos estiloides alargados de más de $40 \mathrm{~mm}$ (2). Así, la variación en la incidencia es notable, se ha informado que se sitúa entre el 1,4 y el 30\%, $y$ afecta mayormente a personas adultas y mujeres (5).

\section{Metodología}

Se realizó una revisión de la literatura exponiendo los conceptos clásicos del síndrome de Eagle y resaltando los planes de tratamiento indicados actualmente, además se muestran imágenes clínicas de una paciente atendida en el Servicio de Cirugía Oral y Maxilofacial del Hospital Militar Central de Bogotá. 


\section{Etiología}

Se ha sugerido que la calcificación es de origen ectópico, pero prevalece en pacientes con enfermedad renal en etapa terminal, alteraciones en los niveles de calcio y potasio y el metabolismo de la vitamina D. También se ha reportado que se encuentra después de la amigdalectomía o traumas (5).

Diversas teorías describen el origen congénito del proceso de alargamiento de la estiloides asociado con la persistencia del cartílago. También se considera la calcificación del ligamento estiloideo por un proceso desconocido y asimismo se sugiere el crecimiento de tejido óseo en la inserción del ligamento estiloideo.

Steinmann propuso otra lista de teorías para explicar la osificación: 1) la teoría de la hiperplasia reactiva que se da cuando el trauma activa los restos de células conectivas y fibrocartilaginosas; 2) la teoría de reactividad metaplásica, una recuperación anormal posterior a un trauma en el ligamento estiloideo que se ve reflejado en osificación; 3) la teoría de la diferencia anatómica sin antecedente de trauma. Camarda propuso una cuarta teoría, en ausencia de calcificación radiográfica evidente, la teoría del desarrollo anormal asociado al envejecimiento en la cual se plantea que la pérdida de la elasticidad de los tejidos blandos promueve una inflamación localizada en la inserción del ligamento estilomandibular (6).

\section{Características clínicas}

El diagnóstico del síndrome de Eagle se logra mediante una cuidadosa recopilación de la historia clínica, evidencia de dolor exacerbante del proceso estiloideo en la fosa amigdalina a la palpación digital y a través de una radiografía panorámica o tomografía computarizada (7).

Se caracteriza por dolor facial, cuello y garganta, posible disfagia y tinnitus. El dolor se puede irradiar al oído ipsilateral en el momento en que se gira la cabeza al lado opuesto mientras simultáneamente deglute (7).

Una forma vascular del síndrome de Eagle es la compresión arterial de la carótida, que se manifiesta en el giro de la cabeza con dolor periorbital y síntomas transitorios de ataques isquémicos o accidentes cerebrovasculares (mareos, cefalea o síncope). El examen clínico del paciente puede permitir palpación del proceso estiloides alargado. La palpación bimanual extraoral e intraoral permite localizar el dolor en la región retromandibular (3) (figura 1).

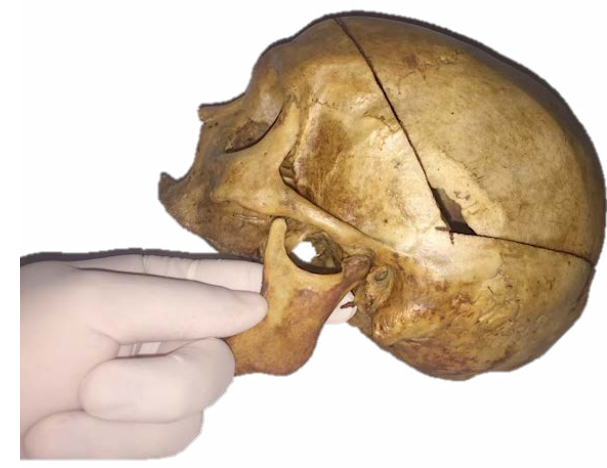

Figura 1. Diagrama de palpación local de la elongación del proceso estiloides (8).

La distribución de los síntomas relacionados se da de la siguiente manera: sintomas funcionales (75\%), garganta (80\%), sensación de cuerpo extraño (55\%), dolor (40\%), otalgia $(40 \%)$, cefalea $(25 \%)$, carotidinia $(20 \%)$, dolor temporomandibular (10\%), neuralgia del glosofaríngeo $(10 \%)$ y dolor facial (5\%) (5).

\section{Por medio de radiografía}

La longitud normal del proceso estiloides va desde 1,52 a $4,77 \mathrm{~cm}$. Keur declaró que, si la porción que aparece en las radiografías osificada es más larga de $30 \mathrm{~mm}$, esto podría considerarse como un proceso alargado. Sin embargo, Thot explicó que la presencia de un proceso estiloideo alargado no suele ser patognomónico para el síndrome de Eagle porque muchos pacientes con esta incidencia radiográfica no presentan hallazgos sintomáticos (6).

\section{Tipos de alargamiento del proceso estiloideo}

Tipo I es un proceso alargado (el más frecuente), tipo II es un proceso seudoarticulado y tipo III un proceso segmentario. El proceso estiloides es considerado "estrecho" si tiene una angulación de $65^{\circ}$, "normal" si el rango de angulación está entre $75^{\circ}$ y "amplio" cuando la angulación es $>75^{\circ}$ (9) (figura 2).
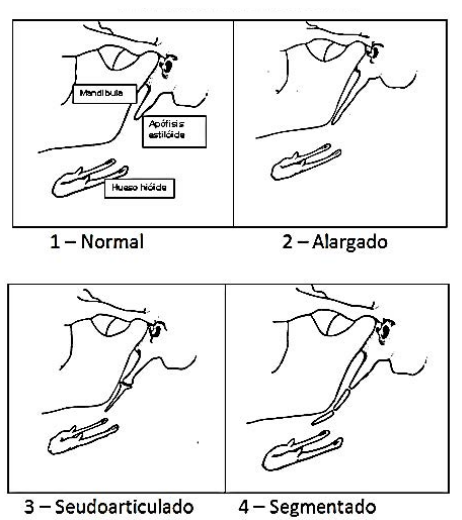

Figura 2. Esquema de clasificación de la apófisis estiloides (9). 
Además, el patrón de osificación es también clasificado: A, contorno calcificado (el más frecuente); B, parcialmente calcificado; $\mathrm{C}$, nodular; $\mathrm{D}$, completamente calcificado (8).

\section{Diagnóstico diferencial}

El diagnóstico diferencial debe incluir todos los trastornos caracterizados por dolor cervicofacial, esfenopalatino o neuralgia glosofaríngea, trastornos temporomandibulares, otitis, odontalgia, mastoiditis, sialolitiasis submandibular o sialoadenitis, tumores de la faringe o base de la lengua, tendinitis temporal, síndrome de Ernest (síndrome del ligamento estilomandibular), cefalea, osteoartritis cervical y síndrome de arteria carótida (10).

\section{Tratamiento}

El síndrome de Eagle tiene un manejo quirúrgico o no quirúrgico, la elección debe ser discutida con el paciente y depende de la gravedad de los síntomas. El enfoque conservador se basa en la tranquilidad del paciente, la administración de analgésicos y las infiltraciones de esteroides. El abordaje quirúrgico puede ser transoral, que es comúnmente aceptado ya que proporciona mejores resultados estéticos, pero tiene la desventaja de la limitada visión intraoperatoria, o extraoral que brinda una mejor visualización del campo quirúrgico y disminución del riesgo de infección en espacios profundos del cuello, sin embargo, genera una cicatriz visible y conlleva aumento del riesgo de daño del nervio facial $(2,8)$.

La planificación virtual, angiografías y el uso del piezo eléctrico intraoperatorio es recomendado por diversos autores puesto que minimiza las complicaciones asociadas a la estrecha relación existente entre el proceso estiloideo y varias ramas de la arteria carótida (8).

\section{Caso clínico}

Paciente femenina de 34 años, sin antecedentes médicos, con sensación de cansancio en el cuello, dolor a la palpación en fosa amigdalina, en maseteros bilateral con predominio del lado izquierdo que se irradia al oído y a nivel cervical ipsilateral de un año de evolución, sin tratamiento hasta el momento.

Clínicamente presentaba orofaringe sin alteraciones, conductos auditivos externos permeables, se descartan trastornos de la articulación temporomandibular que evidencia adecuados movimientos durante apertura y cierre mandibular, sin ruidos o brincos articulares, a su vez se descarta enfermedad odontogénica o glandular observando normalidad en las estructuras dentales y normalidad a la palpación y en función de las glándulas salivales sublingual y submanibular sin inflamación asociada con adecuada permeabilidad de los conductos salivales.

Como primera medida, se solicitan imágenes diagnósticas, radiografía panorámica y tomografía computarizada en cortes coronal, sagital, axial y reconstrucción 3D (figuras $4,5,6,7)$, en las que se evidencia elongación de los procesos estiloideos bilateralmente, medidos en conjunto con el servicio de radiología tras lo cual se obtiene los siguientes valores: $4,6 \mathrm{~cm}$ del lado derecho y del lado izquierdo $4,3 \mathrm{~cm}$.

Así pues, teniendo en cuenta las anteriores medidas y la anatomía observada en las imágenes diagnósticas, comparamos y clasificamos según los tipos de alargamiento del proceso estiloideo mencionados de la siguiente manera: del lado derecho, un proceso segmentario, y del lado izquierdo, un proceso pseudoarticulado.

El plan inicial con la paciente fue realizar un manejo conservador mediante terapia física localizada y farmacológica establecida por fisiatría, servicio al cual fue remitida; con el manejo dado por este servicio, la paciente presenta una evolución favorable con resolución parcial de la sintomatología, motivo por el cual se descarta un manejo quirúrgico sopesando riesgo-beneficio (figura 3).

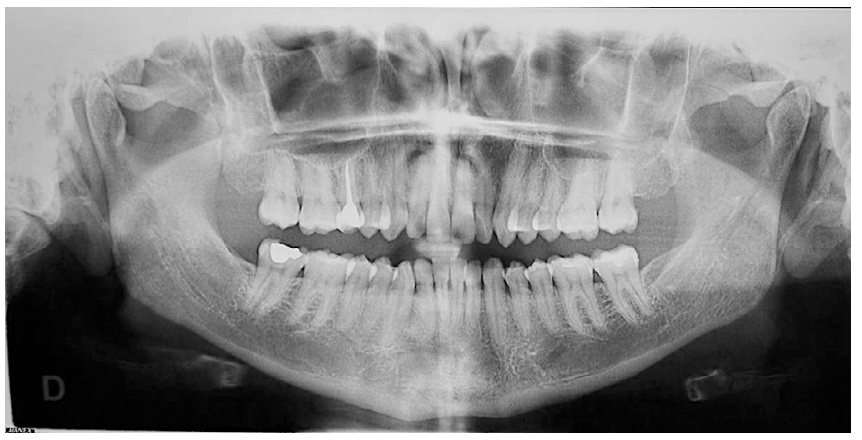

Figura 3. Radiografía panorámica. 


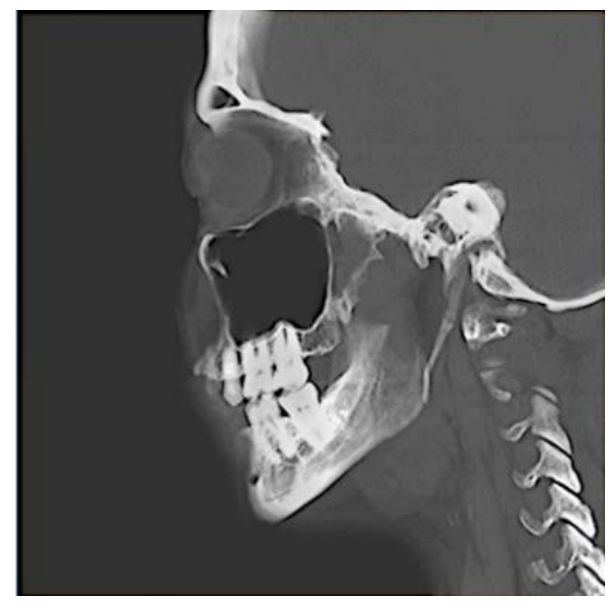

Figura 4. TC corte sagital derecho e izquierdo.

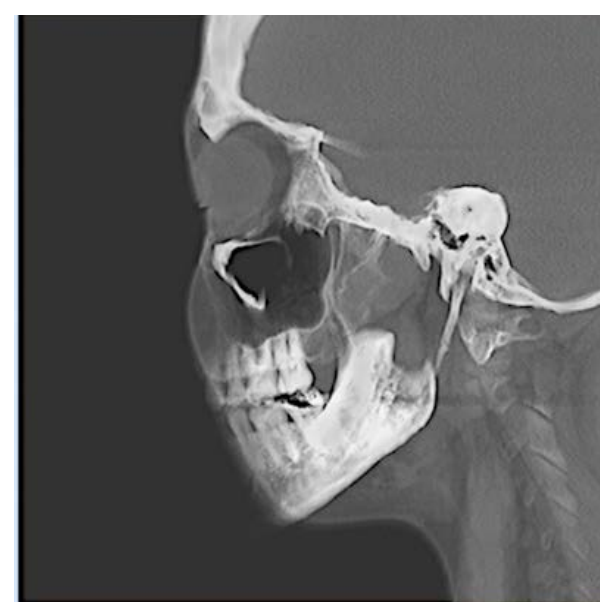

Figura 5. TC corte coronal.

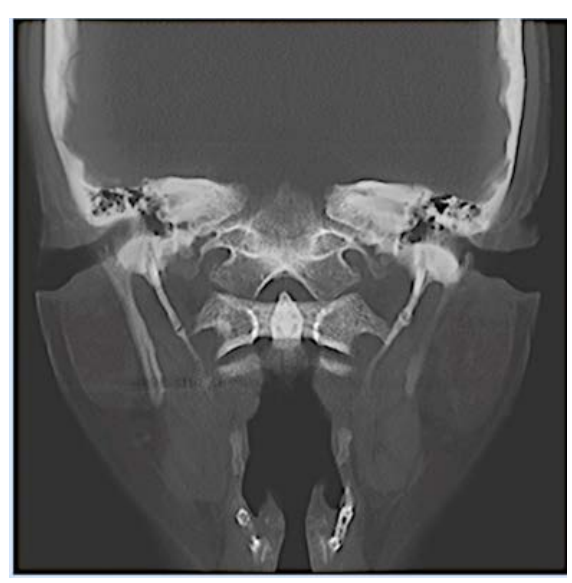

Figura 6. TC corte axial.
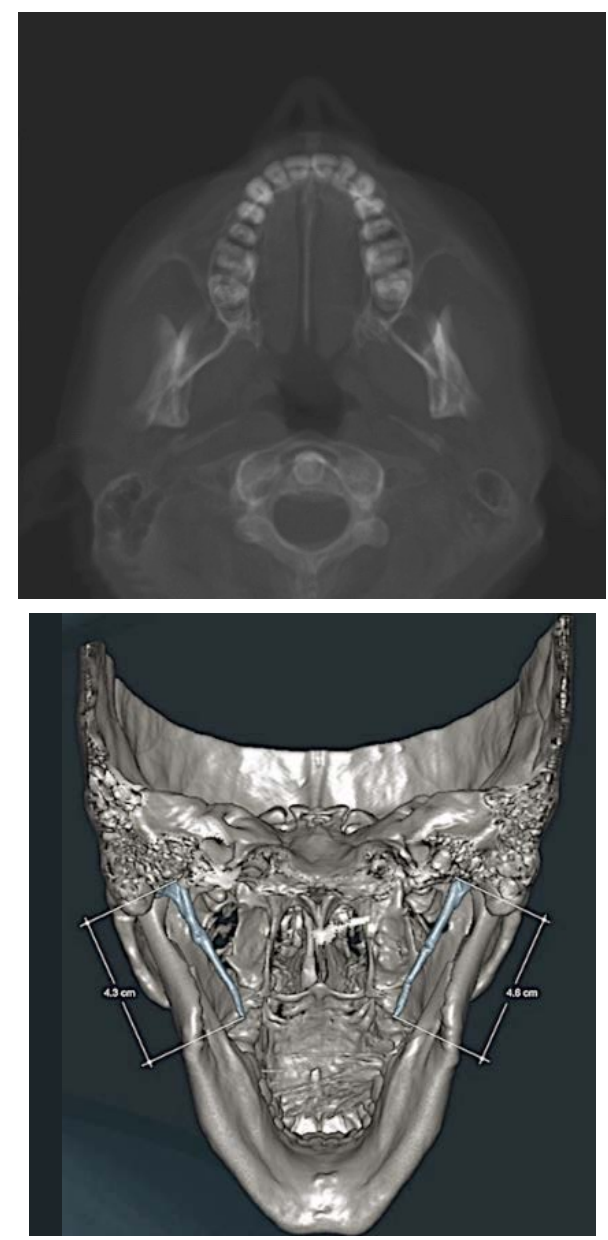

Figura 7. TC con reconstrucción $3 D$ en que se observa calcificación de la apófisis estiloides tipo segmentada.

Fuente: Servicio de Cirugía Oral y Maxilofacial del Hospital Militar Central de Bogotá.

\section{Discusión}

El síndrome de Eagle se considera como la elongación del proceso estiloides el cual se extiende entre el hueso temporal y el hioides, con osificación del ligamento estiloideo o sin ella.

Presenta variedad de signos y síntomas como en el caso anteriormente presentado: dolor, sensación de cuerpo extraño en la faringe, limitaciones en la apertura bucal, dificultad con la fonación, disfagia, otalgia, tinnitus, trismo, dolor cervical, movilidad reducida del cuello. También se puede confundir fácilmente con el dolor dental, trastornos de las articulaciones o la función temporomandibular, por eso es importante determinar los diagnósticos diferenciales de esta enfermedad. 
Los pacientes pueden ser tratados de forma conservadora o quirúrgicamente. El manejo conservador incluye fisioterapia, infiltración con anestésicos o corticosteroides y el tratamiento con fármacos antiinflamatorios, anticonvulsivos o antidepresivos. El manejo quirúrgico consiste en fracturar el proceso estiloides bajo anestesia general a través de dos principales abordajes quirúrgicos, transoral o transcervical. Cada uno tiene sus propias ventajas y desventajas, sin embargo, consideramos que el manejo inicial siempre debe estar dado por el tratamiento menos invasivo o "conservador" como en el caso anteriormente descrito, evitando riesgos, costos y posible compromiso estético por los abordajes utilizados en un manejo quirúrgico, sin descartar este en caso de no tener resolución de la sintomatología.

\section{Conclusión}

El síndrome de Eagle debe contemplarse dentro de los diagnósticos diferenciales en pacientes con dolor cervicofacial crónico. La realización de una adecuada historia clínica, al igual que un exhaustivo examen clínico, ayudan a determinar los signos y síntomas clásicos, aunque variados de esta enfermedad, de igual forma el uso de imágenes diagnósticas ayudan a determinar el tipo de alargamiento del proceso estiloideo. El tratamiento quirúrgico dependerá principalmente de las manifestaciones clínicas, su severidad y la respuesta negativa al tratamiento conservador.

\section{Referencias}

1. Costantinides F, Vidoni G, Tonni I, et al. Orofacial pain induced by Eagle syndrome in an elderly patient with temporomandibular disorders-a case report. Gerodontology. 2016; 33(3):428-431.

2. Spalthoff S, Zimmerer R, Dittmann O, et al. Piezoelectric surgery and navigation: a safe approach for complex cases of Eagle syndrome. Int $\mathrm{J}$ Oral Maxillofac Surg. 2016;45(10): 1261-1267.

3. Al Weteid AS, Miloro M. Transoral endoscopic-assisted styloidectomy: How should Eagle syndrome be managed surgically? Int J Oral Maxillofac Surg. 2015;44(9):11811187.

4. Eagle WW. Symptomatic elongated styloid process report of two cases of styloid process-carotid artery syndrome with operation. Arch Otolaryngol Head Neck Surg. 1949;49(5): 490-503.

5. Martins WD, de Oliveira Ribas M, Bisinelli J, et al. Eagle's syndrome: treatment by intraoral bilateral resection of the ossified stylohyoid ligament. A review and report of two cases. Cranio. 2013;31(3):226-232.

6. Costantinides F, Vidoni G, Bodin C, et al. Eagle's syndrome: signs and symptoms. Cranio. 2013;31(1):56-60.

7. Bertossi D, Albanese M, Chiarini L, et al. Eagle syndrome surgical treatment with piezosurgery. J Craniofac Surg. 2014;25(3), 811-813.

8. Kiralj A, Ilić M, Pejaković B, et al. Eagle's syndrome: a report of two cases. Vojnosanit Pregl. 2015;72(5):458-462.

9. Ragone Guimarães SM, Cherfên Peixoto B, Batista Gomes $\mathrm{M}$, et al. Estudio radiográfico de las alteraciones morfológicas de la apófisis estiloide y de la rotación del cóndilo mandibular en pacientes con desorden temporomandibular. Acta Odontol Venez, 2010;48(1):87-92.

10. Kent DT, Rath TJ, Snyderman C. Conventional and 3-dimensional computerized tomography in Eagle's syndrome, glossopharyngeal neuralgia, and asymptomatic controls. Acta Otolaryngol. 2015;153(1):41-47. 